\title{
Absolute and convective instabilities of parallel propagating circularly polarized Alfvén waves: numerical results
}

\author{
D. Simpson, M. S. Ruderman, and R. Erdélyi
}

\begin{abstract}
Department of Applied Mathematics, University of Sheffield, Hicks Building, Hounsfield Road, Sheffield S3 7RH, UK
\end{abstract}
Received 8 June 2005 / Accepted 27 February 2006

\section{ABSTRACT}

\begin{abstract}
Context. The stability of parallel propagating circularly polarized Alfvén waves (pump waves) has been studied for more than four decades with the use of normal mode analysis. It is well known that the normal mode analysis does not answer the question if a pump wave looks stable or unstable in a particular reference frame. To answer this question it is necessary to find out if the instability is absolute or convective in this reference frame.

Aims. We extend our previous study of absolute and convective instabilities of pump waves with small amplitude to pump waves with arbitrary amplitude.

Methods. To study the absolute and convective instabilities of pump waves with arbitrary amplitude we numerically implement Brigg's method.

Results. We show that the wave is absolutely unstable in a reference frame moving with the velocity $U$ with respect to the rest plasma if $U$ satisfies the inequality $U_{l}<U<U_{r}$ and convectively unstable otherwise, and calculate the dependences of $U_{l}$ and $U_{r}$ on the dimensionless wave amplitude $a$. We also calculate the dependence of the increment of the absolute instability on $U$ for different values of $a$. When the instability is convective $\left(U<U_{l}\right.$ or $U>U_{r}$ ) we study the signalling problem. We show that spatially amplifying waves exist only when the signalling frequency is in two symmetric frequency bands, and calculate the dependences of the boundaries of these bands on $U$ for different values of $a$. We also obtain the dependences of the maximum spatial amplification rate on $U$ for different values of $a$. The implication of these results on the interpretation of observational data from space missions is discussed. In particular, it is shown that circularly polarized Alfvén waves propagating in the solar wind are convectively unstable in a reference frame of any realistic spacecraft.
\end{abstract}

Key words. plasmas - magnetohydrodynamics (MHD) - waves - instabilities - solar wind

\section{Introduction}

Finite amplitude circularly polarized parallel propagating Alfvén waves are exact solutions of the nonlinear magnetohydrodynamic (MHD) equations. The stability of these waves has been studied for more than four decades. Galeev \& Oraevskii (1963) studied the stability of these waves under the assumption that the wave amplitude and the plasma $\beta$ are small (see also Sagdeev \& Galeev 1969). Derby (1978) and Goldstein (1978) studied the stability of circularly polarized Alfvén waves (pump waves) with arbitrary amplitudes in finite $\beta$ plasmas. Sakai \& Sonnerup (1983), Longtin \& Sonnerup (1986), Wong \& Goldstein (1986) and Brodin \& Stenflo (1988) investigated the stability of pump waves using the two fluid description. Viñas \& Goldstein (1991) studied the linear stability of pump waves with respect to obliquely propagating perturbations. Ghosh et al. $(1993,1994)$ and Ghosh \& Goldstein (1994) used numerical simulations to analyse the nonlinear evolution of circularly polarized Alfvén waves in two dimensions. Hollweg et al. (1993) and Jayanti \& Hollweg (1994) analyzed the stability of circularly polarized Alfvén waves in a plasma with streaming $\mathrm{He}^{++}$ions. The kinetic description for studying the stability of circularly polarized Alfvén waves was used by Ling \& Abraham-Srauner (1979), Spangler $(1989,1990)$ and Inhester (1990). Low (1996) studied the stability of circularly polarized Alfvén waves in a selfgravitating plasma. A comparison of theory and observations near the Earth's bow shock was given by Spangler (1997).
Among recent publications we have to note the following. Del Zanna et al. $(2001,2003)$ and Del Zanna \& Velli (2002) developed a three-dimensional MHD code specially designed to study the stability and nonlinear evolution of Alfvén waves. They applied their numerical results to the evolution of Alfvén wave spectra in the solar wind (Del Zanna et al. 2001, 2003) and to plasma heating in coronal holes (Del Zanna \& Velli 2002). Shevchenko et al. (2003) used the Derivative Nonlinear Schrödinger equation (DNLS) to study the parametric decay instability of Alfvén packets propagating in opposite directions. Hertzberg et al. (2003, 2004a,b) and Cramer et al. (2003) extended the linear theory of parametric instabilities of Alfvén waves to multicomponent and dusty plasmas. Matsukiyo \& Hada (2003) studied the parametric instabilities of circularly polarized Alfvén waves in a relativistic electron-positron plasma.

It is well known that the normal mode analysis is not sufficient to conclude whether a stationary state, homogeneous in at least one spatial direction, appears stable or unstable in a fixed reference frame. The reason is that the normal mode analysis deals with spatially periodic perturbations, while real perturbations are bounded in space. To determine if the stationary state appears stable or unstable in a fixed reference frame we have to solve the initial value problem for spatially bounded perturbations and then evaluate the asymptotic behaviour of the solution when the time tends to infinity. Two scenarios are then possible. In the first scenario the initial perturbation grows exponentially with time at any fixed spatial position. This situation is referred 
to as "absolute" instability. In the second scenario the initial perturbation grows exponentially with time, but simultaneously it is convected out of any finite portion of the spatial domain so fast that eventually it decays exponentially at any fixed spatial position. This situation is referred to as a "convective" instability.

The concept of absolute and convective instabilities was first developed in plasma physics (Briggs 1964; Bers 1973). Later it was applied to hydrodynamic stability problems, in particular, to stability of geophysical and astrophysical flows (Kulikovskii \& Shikina 1977; Huerre \& Monkewitz 1985; Brevdo 1988; Ruderman 2000; Wright et al. 2000, 2002; Mills et al. 2000; Terra-Homem \& Erdélyi 2003, 2004; Ruderman et al. 2004). Recently Ruderman \& Simpson (2004b) and Simpson \& Ruderman (2005) (Papers I and II in what follows) studied the absolute and convective instabilities of circularly polarized Alfvén waves analytically using the dimensionless pump wave amplitude $a$ as a small parameter. In this paper we use numerical technics to extend their analysis for arbitrary $a$. The paper is organized as follows. In the next section we give a very brief description of Briggs' method for studying the absolute and convective instabilities, and present the numerical results of our analysis of the absolute and convective instabilities. In Sect. 3 we investigate spatially amplifying waves. In Sect. 4 we discuss possible implications of our results on the interpretation of observational data obtained onboard of space missions. Section 5 contains the summary of results and our conclusions.

\section{Absolute and convective instabilities}

We study the parametric instability of a circularly polarized Alfvén wave propagating parallel to an ambient magnetic field using the equations of ideal magnetohydrodynamics. The stability of this wave is governed by the following dispersion equation (Derby 1978; Goldstein 1978; Ruderman \& Simpson 2004a):

$$
\begin{aligned}
D(\omega, k) \equiv & \left(\omega^{2}-b^{2} k^{2}\right)(\omega-k)\left[(\omega+k)^{2}-4\right] \\
& -a^{2} k^{2}\left(\omega^{3}+\omega^{2} k-3 \omega+k\right)=0,
\end{aligned}
$$

where $\omega$ and $k$ are the frequency and wavenumber of the perturbation, non-dimensionalised by the corresponding pump wave quantities, $b=c_{\mathrm{S}} / v_{\mathrm{A}}, c_{\mathrm{S}}$ is the sound speed and $v_{\mathrm{A}}$ is the Alfvén speed. The question which we address in this paper is whether unstable modes described by this dispersion equation appear unstable to an observer moving with the velocity $\bar{U}$ with respect to the rest plasma parallel to the ambient magnetic field, which is in the $x$-direction in Cartesian coordinates $x, y, z$. We use Briggs' method in the same way as in Papers I and II, but we generalize the analysis to pump waves with arbitrary $a$. The details of Briggs' method can be found, e.g., in Briggs (1964) and Bers (1973). A brief description of Brigg's method in application to the absolute and convective instabilities of circularly polarized Alfvén waves is given in Papers I and II. Hence, here we do not describe this method and only mention that it is based on evaluating the asymptotic behaviour of perturbations as $t \rightarrow \infty$. The perturbation of the density is given by the inverse FourierLaplace integral of the form

$\delta \rho(x, t)=\int_{i \tau-\infty}^{i \tau+\infty} \mathrm{e}^{-\mathrm{i} \omega t} \mathrm{~d} \omega \int_{-\infty}^{\infty} \frac{T(k, \omega)}{\widetilde{D}(k, \omega)} \mathrm{e}^{\mathrm{i} k x} \mathrm{~d} k$.

The perturbation of any other quantity is given by a similar expression. The function $T(k, \omega)$ is determined by initial conditions and it is not important for the asymptotic response; $\widetilde{D}(k, \omega)=D(k, \widetilde{\omega})$, where $\widetilde{\omega}=\omega+k U$ is the Doppler shifted frequency, $U=\bar{U} / v_{\mathrm{A}}$, and $\tau$ is a quantity taken to be larger that the

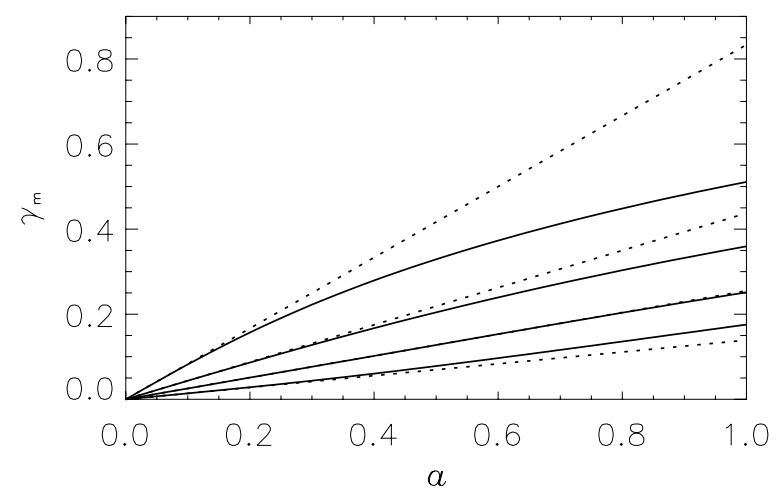

Fig. 1. Maximum growth rate $\gamma_{M}$ versus $a$ for the decay instability. Solid lines represent numerical results for $b=0.2$ (top line) in increments of 0.2 up to $b=0.8$ (bottom line). The corresponding analytical results obtained with the use of the small amplitude approximation are shown by dotted lines. Note that the analytic small amplitude approximation and the numerical results almost coincide for $b=0.6$.

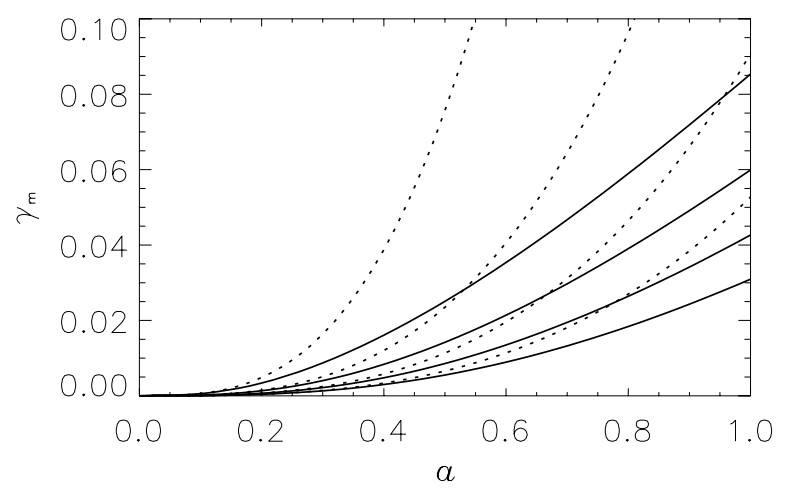

Fig. 2. The same as Fig. 1 but for the beat instability with $b=1.2$ (top line) in increments of 0.2 up to $b=1.8$ (bottom line). Note that the analytic results agree better with the numerical results as $b$ increases.

maximum imaginary part of $\omega$ for real $k$ determined by Eq. (1), i.e. the maximum growth rate of the instability $\gamma_{M}$ calculated using the normal mode analysis.

Now we present the results of the absolute and convective instability analysis for pump waves of arbitrary amplitude. We stated earlier that this research is motivated by the application of the results to Alfvén waves in the solar wind. With this in mind we will restrict our analysis to pump wave amplitudes less than unity $(a<1)$ and, in the case of the beat instability $(b>1)$, we only consider $b$ to be of the order of unity.

We start by analyzing the effect of large pump wave amplitude on the maximum growth rates of the decay and beat instabilities. Similar results have been presented in Jayanti \& Hollweg (1993), but we briefly recall them here as we frequently refer to these results in what follows. In Figs. 1 and 2 we plot $\gamma_{M}$ as a function of $a$ for the decay and beat instabilities respectively. In Fig. 1 we see that the analytical approximation is definitely good for $a \lesssim 0.2$ and any value of $b$, but, in general, fails for larger values of $a$.

Figure 2 shows that, for the range of values of $b$ considered for the beat instability, the approximation of $\gamma_{\mathrm{M}}$ always gives an overestimate of the exact values. For $b \approx 1$ the error is very large but, as $b$ increases, the error decreases. Once again analytics gives a good approximation for $a \lesssim 0.2$ and any value of $b$. It is easy to see from both figures that increasing $a$ produces a substantial increase in the values of $\gamma_{\mathrm{M}}$, particularly for the beat 

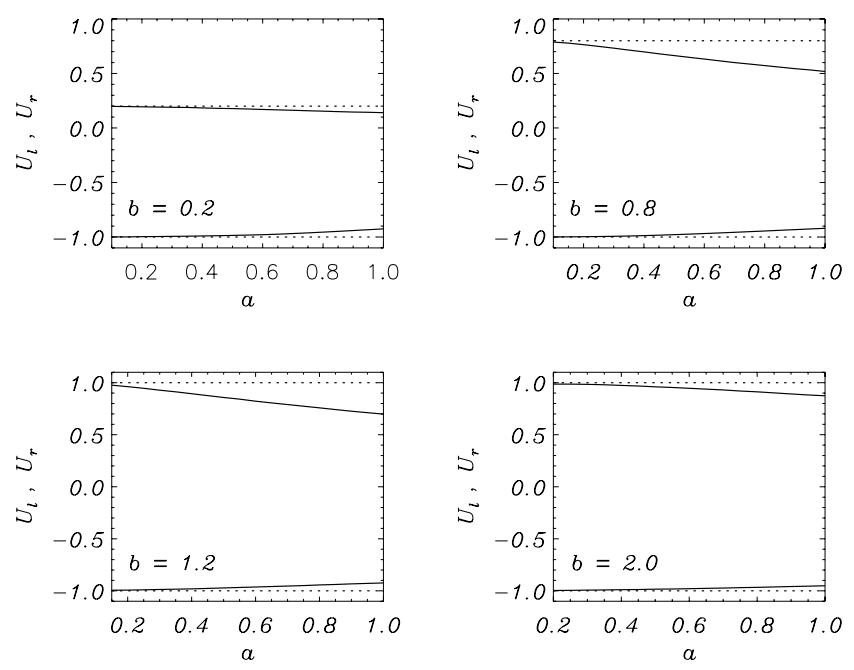

Fig. 3. The solid lines show the numerically calculated dependences of $U_{l}$ (lower curves) and $U_{r}$ (upper curves) on $a$ for $b=0.2,0.8$, corresponding to the decay instability, and $b=1.2,2.0$, corresponding to the beat instability. The dotted lines show the analytical approximation for small $a$.

instability where $\gamma_{\mathrm{M}}$ is proportional to $a^{3}$ for small $a$ (Jayanti \& Hollweg 1993).

Note that both for the decay and beat instabilities $\gamma_{M}$ decreases when $b$ increases. This is a well-known result (see, e.g., Galeev \& Oraevskii 1963; Jayanti \& Hollweg 1993).

Now we proceed to the results of the absolute and convective instability analysis. We made numerical calculations for four values of $b: b=0.2,0.8,1.2$ and 2.0. In Fig. 3 we show the dependences of the boundaries of the absolute instability, $U_{l}$ and $U_{r}$, on $a$ for $0<a<1$. We see that the interval of values of $U$ corresponding to the absolute instability becomes narrower as $a$ increases.

The asymptotic behaviour of perturbation of any quantity is proportional to $t^{-1 / 2} \exp \left(\mathrm{i} k_{m} x-\mathrm{i} \omega_{m} t\right)$, where $k_{m}=k_{m r}+\mathrm{i} k_{m i}$ and $\omega_{m}=\omega_{m r}+\mathrm{i} \omega_{m i}$. The quantity $\omega_{m i}$ gives the perturbation growth rate in a reference frame moving with the dimensionless velocity $U$ with respect to the rest plasma. The quantity $\omega_{m r}$ is the oscillatory frequency of the growing wave packet. The quantities $-k_{m i}$ and $k_{m r}$ give the local spatial amplification rate and the wave number across the wave packet, respectively. The dependences of $\omega_{m i}, \omega_{m r}, k_{m i}$ and $k_{m r}$ on $U$ for different values of $a$ and $b$ are shown in Figs. 4-7.

In Papers I and II we found that, for small $a$, the plots of $\omega_{m i}, \omega_{m r}, k_{m i}$ and $k_{m r}$ versus $U$ should have the form shown in Fig. 8. Note that $\omega_{m i}$ takes its maximum value, $\gamma_{\mathrm{M}}$, at $U=U_{\mathrm{M}}=$ $(b-1) / 2$ for the decay instability and at $U=U_{\mathrm{M}}=0$ for the beat instability. $\mathfrak{R}\left(\omega_{m}\right)$ is represented by a straight line which starts at $\mathfrak{R}\left(\omega_{m}\right)=2$ when $U=-1$ both for the decay and beat instability and decreases to $\mathfrak{R}\left(\omega_{m}\right)=0$ when $U=b$ for the decay instability or $U=1$ for the beat instability. $\mathfrak{J}\left(k_{m}\right)=0$ when $U=U_{\mathrm{M}}$. Finally, $\mathfrak{R}\left(k_{m}\right)$ as a function of $U$ is given by a horizontal line $\mathfrak{R}\left(k_{m}\right)=2 /(1+b)$ for the decay instability and $\mathfrak{R}\left(k_{m}\right)=1$ for the beat instability. It is worth noting that here we have plotted the values of $\omega_{m i}, \omega_{m r}, k_{m i}$ and $k_{m r}$ for $-1<U<b$, but in fact our expressions are only valid for $-1+O(a)<U<$ $b+O(a)$ for the decay instability and for $-1+O(a)<U<$ $1+O(a)$ for the beat instability. This means that within an order of $a$ from the boundaries we do not expect the behavior of the quantities plotted to be very accurate. Comparing with Figs. 4-7 we can see that, as expected, the difference between the analytic
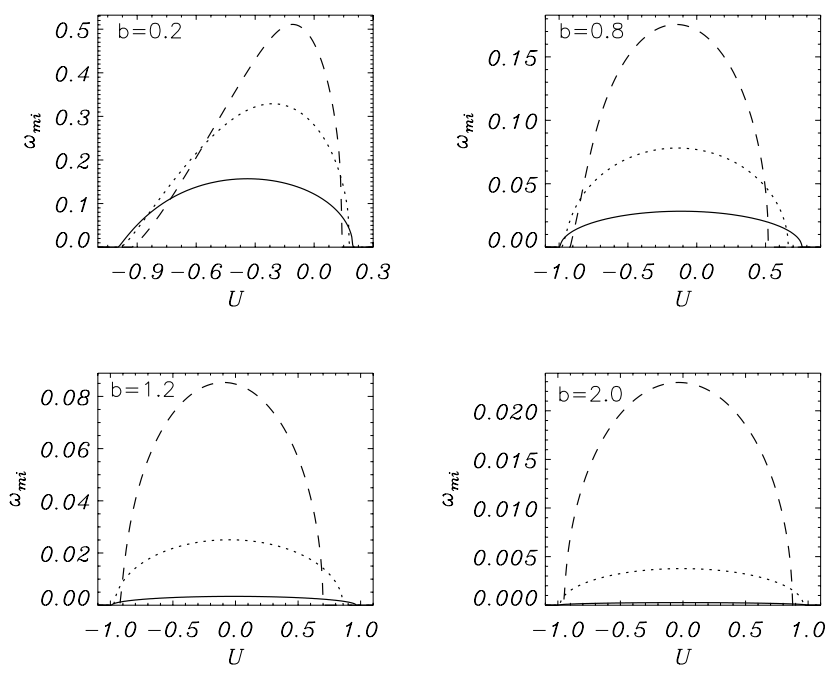

Fig. 4. The growth rate of the absolute instability, $\omega_{m i}$, as a function of $U$ for $a=0.2$ (solid line), $a=0.5$ (dotted line) and $a=1$ (dashed line). Each panel represents a different value of $b$.
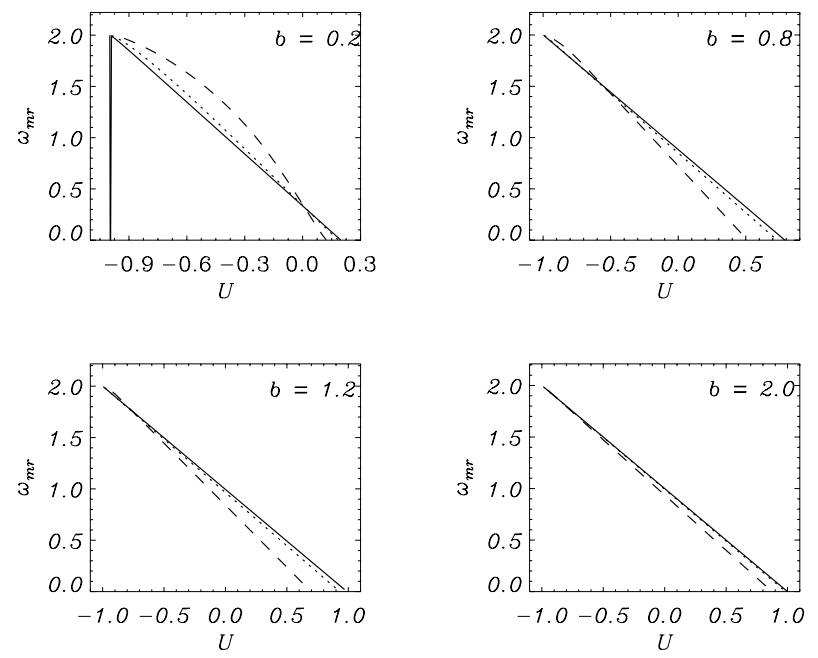

Fig. 5. The same as Fig. 4 but for the oscillatory frequency of the fastest growing mode $\omega_{m r}$.

approximation and the numerical results becomes bigger as $a$ increases.

\section{Spatially amplifying waves}

When the instability is convective, spatially amplifying waves can be driven by a localized harmonic driver. For a detailed description of the method for studying spatially amplifying waves we once again refer the readers to Brigg's (1964). The application of this method to circularly polarized Alfvén waves is described in Papers I and II. Hence, here we do not give the description of this method. We only mention that it is based on evaluating the asymptotic behaviour of perturbations caused by a localized driver as $t \rightarrow \infty$, and then either $x \rightarrow \infty$ or $x \rightarrow-\infty$. The perturbation of the density is given by the inverse FourierLaplace integral of the form

$\delta \rho(x, t)=\int_{i \tau-\infty}^{i \tau+\infty} \frac{\mathrm{e}^{-\mathrm{i} \omega_{\mathrm{d}} t}}{\omega-\omega_{\mathrm{d}}} \mathrm{d} \omega \int_{-\infty}^{\infty} \frac{S(\omega, k)}{\widetilde{D}(\omega, k)} \mathrm{e}^{\mathrm{i} k x} \mathrm{~d} k$. 

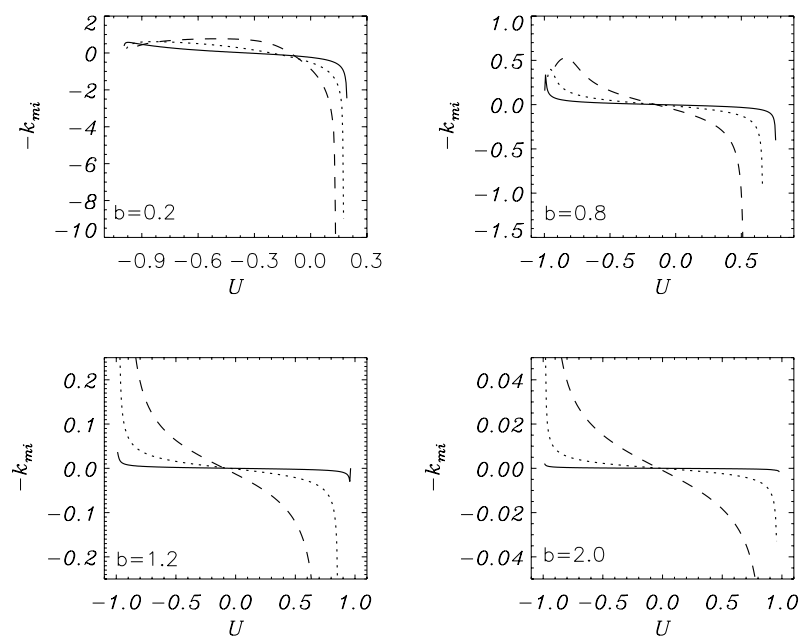

Fig. 6. The same as Fig. 4 but for the local spatial amplification rate $-k_{m i}$.
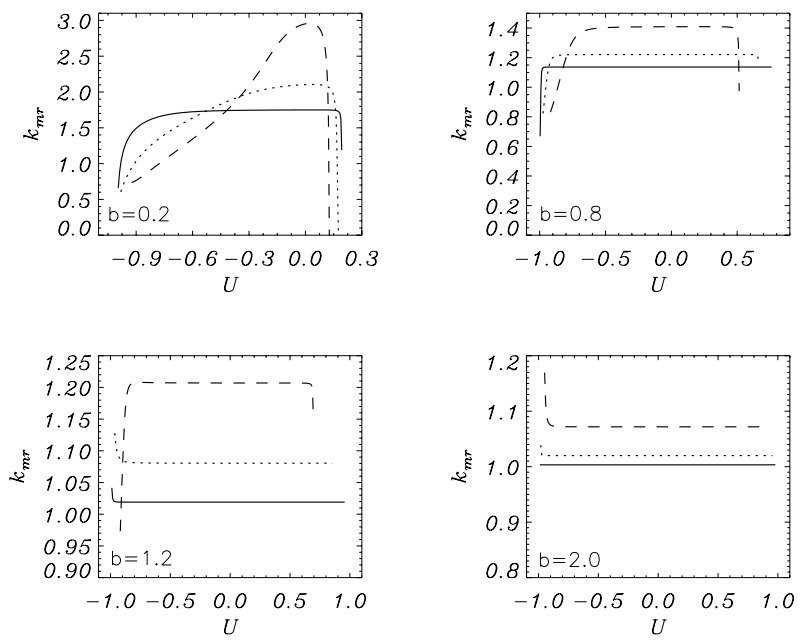

Fig. 7. The same as Fig. 4 but for the wavenumber across the wave packet $k_{m r}$.

Here $S(\omega, k)$ is an analytic function of $k$ and $\omega$ determined by the initial conditions, $\omega_{\mathrm{d}}$ is the (real) driving frequency, $\tau>\gamma_{\mathrm{M}}$, and $\widetilde{D}(\omega, k)$ was introduced in Sect. 2 .

We carried out the numerical analysis for $b=$ $0.2,0.8,1.2$, and 2.0. First of all, we recovered the result obtained analytically in Papers I and II that there are spatially amplifying waves travelling in the negative $x$-direction when $U>U_{r}$ and in the positive $x$-direction for $U<U_{l}$. We also found in Papers I and II that spatially amplifying waves can be driven only when $\omega_{\mathrm{d}}$ is in one of two intervals symmetric with respect to the point $\omega_{\mathrm{d}}=0$. In Fig. 9 the numerically calculated dependences of the boundaries of these intervals, $\omega_{\mathrm{d} 1}$ and $\omega_{\mathrm{d} 2}$, on $U$ are shown for $a=0.2$ and different values of $b$. Since the intervals are symmetric, we considered only the interval with $\omega_{\mathrm{d}}>0$. We see that the analytical and numerical results agree very well. We also carried out the calculations for $a=0.5$ and $a=1.0$, and found that the agreement between the analytical and numerical results is fairly bad for $a=0.5$, and very bad for $a=1.0$, as should be expected.

It is worth noting that, for $a=0.2$ and $b=2.0$, we were only able to carry out the numerical calculations for $|U| \leq 6$ due to the fact that our program cannot compute the roots of $\widetilde{D}(\omega, k)=0$
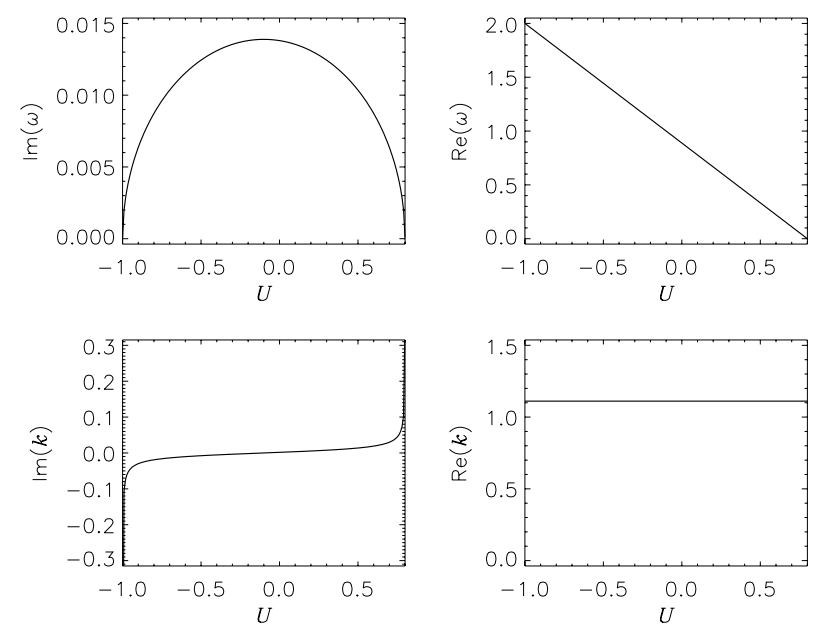

Fig. 8. Plots of $\mathfrak{I}\left(\omega_{m}\right), \mathfrak{R}\left(\omega_{m}\right), \mathfrak{I}\left(k_{m}\right)$ and $\mathfrak{R}\left(k_{m}\right)$ as functions of $U$ given by the analytic theory. The parameter values used are $a=0.1$ and $b=0.8$.
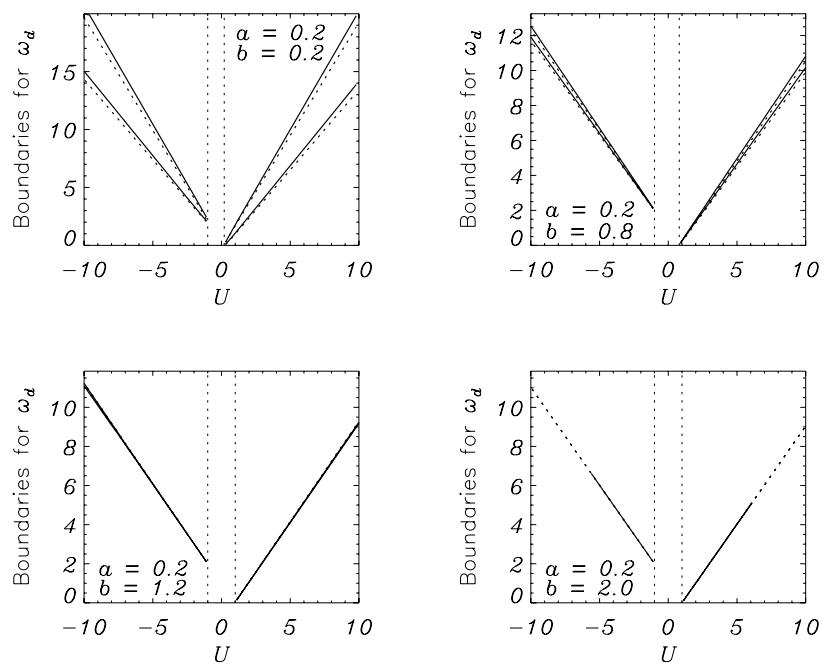

Fig. 9. The boundaries of $\omega_{\mathrm{d}}$ corresponding to spatially amplifying waves as functions of $U$ for $a=0.2$. The dotted lines correspond to the analytical approximation and the solid lines to the numerical solution. The regions in the centres of the plots bounded by two vertical dotted lines correspond to the analytically calculated regions of absolute instability. Note that the numerical calculations for the plot in the bottom right panel have only been carried out for $|U|<6$ due to limitations of the numerical code. Also note that, for the beat instability $(b>1)$, the lower and upper boundaries practically coincide.

to a sufficient accuracy to find the complex $k$ roots for real $\omega$ for $|U|>6$.

In Fig. 10 the spatial amplification rate of waves propagating in the negative $x$-direction, $\mathfrak{J}\left(k_{l}\right)$, is shown as a function of $\omega_{\mathrm{d}}$ for $b=0.8, U=6$ and for three different values of $a$. The dependences of $\mathfrak{J}\left(k_{l}\right)$ on $\omega_{\mathrm{d}}$ for different values of $b$ and $U$ are similar to those plotted in this figure. The same is true for the dependences of the spatial amplification rate of waves propagating in the positive $x$-direction, $-\mathfrak{J}\left(k_{r}\right)$, on $\omega_{\mathrm{d}}$ for different values of $b$ and $U$.

Figure 11 shows the maximum spatial amplification rate $\left(\mathfrak{J}\left(k_{l}\right)\right.$ for $U<U_{l}$ and $-\mathfrak{J}\left(k_{r}\right)$ for $\left.U>U_{r}\right)$ as a function of $U$ for $b=0.2$ and different values of $a$. We can see that the analytical theory gives a very good approximation for $a=0.2$, while the difference between the analytical and numerical results is fairly large for $a=0.5$, and very large for $a=1.0$. We obtained similar 


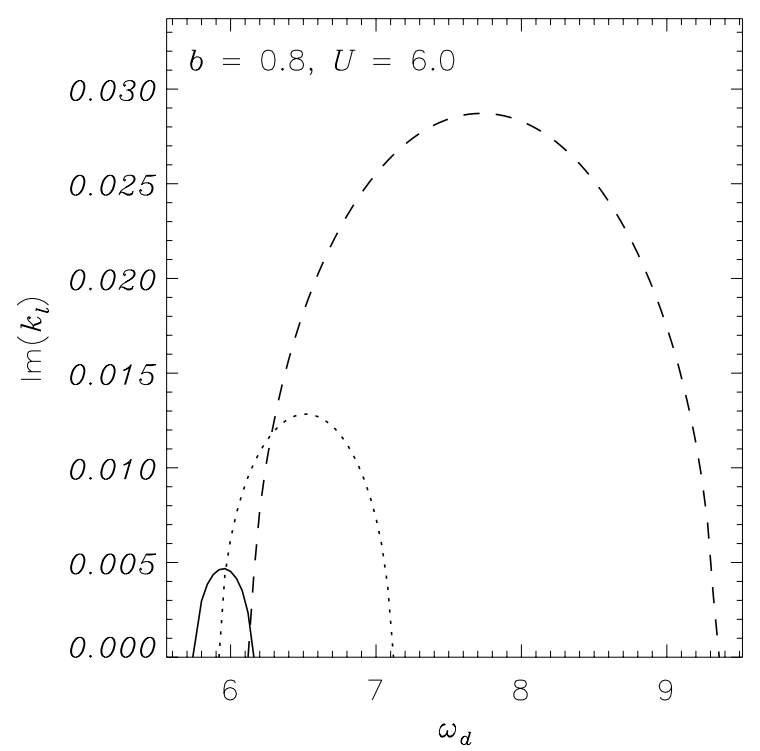

Fig. 10. The spatial amplification rate of waves propagating in the negative $x$-direction, $\mathfrak{J}\left(k_{l}\right)$, is shown as a function of $\omega_{\mathrm{d}}$ for $b=0.8$ and $U=6$. The solid, dotted and dashed lines correspond to $a=0.2, a=0.5$ and $a=1$, respectively.

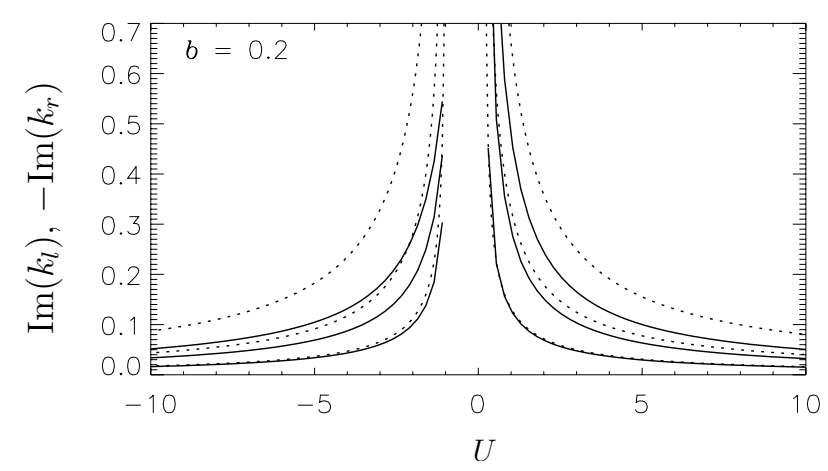

Fig. 11. The maximum spatial amplification rate $\left(\mathfrak{I}\left(k_{l}\right)\right.$ for $U<U_{l}$ and $-\mathfrak{J}\left(k_{r}\right)$ for $\left.U>U_{r}\right)$ as a function of $U$ for $b=0.2$. Dotted lines correspond to the analytical approximation and solid lines to the numerical results. For both the analytical and numerical curves the bottom lines correspond to $a=0.2$, the middle lines to $a=0.5$, and the top lines to $a=1.0$.

results for $b=0.8, b=1.2$ and $b=2$, although the agreement between the analytical and numerical results for large values of $a$ is better for $b=0.8$ and $b=2$ then for $b=0.2$ and $b=1.2$.

\section{Application to Alfvén waves propagating in the solar wind}

In this section we consider a possible implication of the results of this paper on the interpretation of observational data on Alfvén wave instability obtained onboard of space missions. Alfvén waves propagating in the solar wind plasma have been observed during various space missions (e.g., Belcher \& Davis 1971; Tu $\&$ Marsch 1995). It follows from the results presented in Sect. 2 that a circularly polarized Alfvén wave is convectively unstable in any reference frame moving with respect to the rest plasma with the speed larger than the Alfvén speed $v_{\mathrm{A}}$. At large enough distances from the sun (say, beyond the Mercury orbit) the speed of the solar wind is much larger than both the Alfvén speed and the speed of any spacecraft. This implies that the speed of the spacecraft reference frame with respect to the rest plasma, which is the solar wind plasma, is much larger than the Alfvén speed. Consequently, the Alfvén waves are convectively unstable in the reference frame of any spacecraft and their parametric instability cannot be observed directly. This result is, of course, not surprising at all. It is intuitively expected that, due to the high speed of the solar wind any perturbation is swept out of a finite region in the solar reference frame.

The observed values of $b$ in the solar wind at the Earth orbit are usually close to unity. Therefore we consider both the case $b<1$, corresponding to the decay instability, and the case $b>1$, corresponding to the beat instability. Let us consider a perturbation of a circularly polarized Alfvén wave propagating in the solar wind that occurs at a distance from the Sun that is much smaller than $1 \mathrm{au}$. This perturbation will exponentially grow with the increment equal to $\bar{\gamma}_{M}$ and propagate away from the Sun with the speed approximately equal to the solar wind speed, where $\bar{\gamma}_{M}=\bar{\omega} \gamma_{M}$ and $\bar{\omega}$ is the dimensional pump wave frequency. If we take the solar wind speed approximately equal to $500 \mathrm{~km} \mathrm{~s}^{-1}$, then the perturbation arrives at the Earth orbit in about $3 \times 10^{5} \mathrm{~s}$. Then the ratio of the perturbation amplitude at the Earth orbit to its initial amplitude is equal to $\exp \left(3 \times 10^{5} \bar{\omega} \gamma_{\mathrm{M}}\right)$, where $\bar{\omega}$ is measured in $\mathrm{s}^{-1}$. To give an example we consider a pump wave with the period equal to three hours, so that $\bar{\omega} \approx 5.8 \times 10^{-4} \mathrm{~s}^{-1}$ and $3 \times 10^{5} \bar{\omega} \gamma_{\mathrm{M}} \approx 175 \gamma_{\mathrm{M}}$. We also restrict our analysis to small or moderate pump wave amplitudes and assume that $a \lesssim 0.3$. Then it follows from Fig. 2 that, for the beat instability, $\gamma_{M} \leq 0.01$ and the ratio of amplitudes does not exceed 6 . This implies that the perturbation remains small up to the Earth orbit unless the initial perturbation is large enough.

On the other hand, Fig. 1 shows that, for the decay instability, $\gamma_{\mathrm{M}}$ can be as big as 0.2 , which gives the amplitude ratio about $10^{15}$. In general, $\gamma_{\mathrm{M}}$ is a monotonically growing function of $a$ and a monotonically decreasing function of $b$, so that the smaller the plasma $\beta$, the larger the instability increment. Therefore we can expect to observe much higher level of fluctuations related to the parametric instability of Alfvén waves in the low $-\beta$ solar wind than in the high- $\beta$ solar wind.

Let us now consider the signalling problem and once again assume that the pump wave has the period equal to three hours and it propagates in the anti-solar direction with respect to the rest plasma. We also assume that $a=0.2$, and $b=0.2$ for the decay instability, while $b=1.2$ for the beat instability. We take the ratio of the solar wind speed to the Alfvén speed approximately equal to 10 . Then the spacecraft reference frame is moving with the dimensionless velocity $U \approx-10$ with respect to the rest plasma.

First we consider the case $b<1$. Then the instability is decay and, in accordance with Fig. 9, spatially amplifying waves have the dimensionless frequencies between 15 and 20 corresponding to the dimensional frequencies between $8.7 \times 10^{-3} \mathrm{~s}^{-1}$ and $1.16 \times 10^{-2} \mathrm{~s}^{-1}$, and the wave periods between $9 \mathrm{~min}$ and $12 \mathrm{~min}$ for the decay instability. These waves propagate in the anti-solar direction and have the dimensionless maximum spatial amplification rate of the order of 0.01 . For $v_{\mathrm{A}} \approx 50 \mathrm{~km} \mathrm{~s}^{-1}$ we obtain $\bar{k}=\bar{\omega} / v_{\mathrm{A}} \approx 1.16 \times 10^{-8} \mathrm{~m}^{-1}$, so that the dimensional spatial maximum amplification rate is approximately equal to $1.16 \times 10^{-10} \mathrm{~m}^{-1}$. If the driver exciting these waves is at the distance from the sun that is much smaller than $1 \mathrm{au}$, then the ratio of the wave amplitude at 1 au to its amplitude at the driver position is larger than $10^{7}$. This implies that such high-frequency waves can be easily observed at the Earth orbit.

Let us now consider the case $b>1$. Then the instability is beat and, in accordance with Fig. 9, the dimensionless frequencies of spatially amplifying waves for the beat instability are in a 
very narrow interval around $\omega_{\mathrm{d}} \approx 11$, which corresponds to the wave period about $16.4 \mathrm{~min}$. In accordance with our numerical investigation the numerically and analytically calculated spatial amplification rates practically coincide for the considered values of $a, b$ and $U$, so that we can use the analytical expression (Eq. (42) in Simpson \& Ruderman 2005). This expression gives the dimensionless spatial amplification rate approximately equal to $5 \times 10^{-4}$, which corresponds to the dimensional spatial amplification rate approximately equal to $5.8 \times 10^{-12} \mathrm{~m}^{-1}$. Once again, considering the driver a distance from the Sun that is much smaller than 1 au, we obtain that the ratio of the wave amplitude at 1 au to its amplitude at the driver position is about 2.4. Hence, these waves are hardly observable unless the driver amplitude is large enough.

\section{Summary and conclusions}

In this paper we continued to investigate the absolute and convective instabilities of parallel propagating circularly polarized Alfvén waves (pump waves) initiated in Papers I and II, where the problem was studied analytically using the pump wave dimensionless amplitude $a$ as a small parameter. We used a numerical approach to extend their analysis to arbitrary values of $a$. We showed that in a reference frame moving with the velocity $\bar{U}$ with respect to the rest plasma the instability is absolute if $U_{l}<U<U_{r}$ and convective otherwise, where $U=\bar{U} / v_{\mathrm{A}}$, and calculated the dependences of $U_{l}$ and $U_{r}$ on $a$. We found that $U_{l}$ is a monotonically increasing and $U_{r}$ a monotonically decreasing function of $a$. We also calculated the dependence of the growth rate of the absolute instability on $U$ for different values $a$ and $b$, and found that the growth rate increases when $a$ increases and $b$ decreases.

In a reference frame where the instability is convective spatially amplifying waves can be excited by an external driver. These waves propagate in the positive $x$-direction if $U<U_{l}$, and in the negative $x$-direction if $U>U_{r}$. In both cases spatially amplifying waves can be excited only when the dimensionless driver frequency $\omega_{\mathrm{d}}$ is in a finite interval. We calculated the dependences of the boundaries of this interval and the maximum spatial amplification rate on $U$ for different values of $a$ and $b$.

We discussed the implication of our results on the interpretation of observational data obtained onboard of space missions. We concluded that a pump wave propagating in the solar wind is only convectively unstable in a reference frame of any spacecraft. As an example, we considered a pump wave with the period of $3 \mathrm{~h}$. We found that a perturbation imposed at a distance from the Sun much smaller than 1 au can reach a substantial amplitude at the Earth orbit in the case of the decay instability, while its amplitude increases only a few times in the case of beat instability. Hence, we concluded that we can expect a much higher level of fluctuations related to the parametric instability of Alfvén waves in the low- $\beta$ solar wind than in the high- $\beta$ solar wind.

We also considered the signalling problem for this particular pump wave. We found that perturbations with the periods much smaller than $3 \mathrm{~h}$ can be excited by an external driver. Their amplitudes grow by many orders of magnitude before they reach the Earth orbit in the case of the decay instability, and only by a few times in the case of the beat instability.
Acknowledgements. M.S.R. acknowledges the support of the U.K.'s PPARC (Particle Physics and Astronomy Research Council). D.S. acknowledges the support by the University of Sheffield Endowment Fellowship. R.E. if grateful to M. Kéray for patient encouragement and acknowledges the support from the NSF Hungary (OTKA, TO43741).

\section{References}

Belcher, J. W., \& Davis, L. Jr. 1971, J. Geophys. Res., 76, 3534

Bers, A. 1973, in Survey Lectures, Proc Int. Congr., Waves and Instabilities in Plasmas, ed. G. Auer, \& F. Cap (Insbruck, Austria: Institute for Theoretical Physics), B1

Brevdo, L. 1988, Geophys. Astrophys. Fluid Dynam., 40, 1

Briggs, R. J. 1964, Electron-stream interaction with plasmas (Cambridge, MA: MIT press)

Brodin, G., \& Stenflo, L. 1988, Phys. Scr., 37, 89

Cramer, N. F., Hertzberg, M. P., \& Vladimirov, S. V. 2003, Pramana-J. Phys., 61, 1171

Del Zanna, L., \& Velli, M. 2002, Adv. Space. Res., 30, 471

Del Zanna, L., Velli, M., \& Londrillo, P. 2001, A\&A, 367, 705

Del Zanna, L., Velli, M., \& Londrillo, P. 2003, in Solar Wind Ten, ed. M. Velli, R. Bruno, \& F. Malara (American Institute of Physics), 566

Derby, N. F. J. 1978, ApJ, 224, 1013

Galeev, A. A., \& Oraevskii, V. N. 1963, Sov. Phys. Dokl., Engl. Transl., 7, 988

Ghosh, S., \& Goldstein, M. L. 1994, J. Geophys. Res., 99, 13351

Ghosh, S., Viñas, A. F., \& Goldstein, M. L. 1993, J. Geophys. Res., 98, 15561

Ghosh, S., Viñas, A. F., \& Goldstein, M. L. 1994, J. Geophys. Res., 99, 19289

Goldstein, M. L. 1978, ApJ, 219, 700

Hertzberg, M. P., Cramer, N. F., \& Vladimirov, S. V. 2003, Phys. Plasmas, 10, 3160

Hertzberg, M. P., Cramer, N. F., \& Vladimirov, S. V. 2004a, Phys. Rev. E, 69, art. No. 056402

Hertzberg, M. P., Cramer, N. F., \& Vladimirov, S. V. 2004b, J. Geophys. Res., 109, A02103

Hollweg, J. V., Esser, R., \& Jayanti, V. 1993, J. Geophys. Res., 98, 3491

Huerre, P., \& Monkewitz, P. A. 1985, J. Fluid Mech., 159, 151

Inhester, B. 1990, J. Geophys. Res., 95, 10525

Jayanti, V., \& Hollweg, J. V. 1993, J. Geophys. Res., 98, 19049

Jayanti, V., \& Hollweg, J. V. 1994, J. Geophys. Res., 99, 23449

Kulikovskii, A. G., \& Shikina, I. C. 1977, Izv. Akad. Nauk SSSR Mekh. Zhid. Gaza, 5, 46 (English translation: Fluid Dynam. 12, 679)

Ling, K. M., \& Abraham-Shrauner, B. 1979, J. Geophys. Res., 84, 6713

Longtin, M., \& Sonnerup, B. U. Ö. 1986, J. Geophys. Res., 91, 6816

Low, Y.-Q. 1996, MNRAS, 279, L67

Matsukiyo, S., \& Hada, T. 2003, Phys. Rev. E, 67, 046406

Mills, K. J., Longbottom, A. W., Wright, A. N., \& Ruderman, M.S. 2000, J. Geophys. Res., 105, 27685

Ruderman, M. S. 2000, Ap\&SS, 274, 327

Ruderman, M. S., \& Simpson, D. 2004a, J. Plasma. Phys., 70, 143

Ruderman, M. S., \& Simpson, D. 2004b, Phys. Plasmas, 11, 4178

Ruderman, M. S., Brevdo, L., \& Erdélyi, R. 2004, Proc. R. Soc. Lond. A, 460, 847

Sagdeev, R. Z., \& Galeev, A. A. 1969, Non-linear Plasma Theory (New York: Benjamin)

Sakai, J. I., \& Sonnerup, B. U. Ö. 1983, J. Geophys. Res., 88, 9069

Shevchenko, V. I., Sagdeev, R. Z., Galinsky, V. L., \& Medvedev, M. V. 2003, Plasma Phys. Rep., 29, 545

Simpson, D., \& Ruderman, M. S. 2005, Phys. Plasmas, 12, 062103

Spangler, S. R. 1989, Phys. Fluids, B1, 1738

Spangler, S. R. 1990, Phys. Fluids, B2, 407

Spangler, S. R. 1997, in Nonlinear Waves and Chaos in Space Plasmas, ed. T. Hada, \& H. Matsumoto (Tokyo: Terrapub), 171

Terra-Homem, M. T., \& Erdélyi, R. 2003, A\&A, 403, 425

Terra-Homem, M. T., \& Erdélyi, R. 2004, A\&A, 413, 7

Tu, C. Y., \& Marsch, E. 1995, MHD Structures, Waves and Turbulence in the Solar Wind, Observations and Theories (Kluwer)

Viñas, A. F., \& Goldstein, M. L. 1991, J. Plasma. Phys., 46, 129

Wong, H. K., \& Goldstein, M. L. 1986, J. Geophys. Res., 91, 5617

Wright, A. N., Mills, K. J., Ruderman, M. S., \& Brevdo, L. 2000, J. Geophys. Res., 105, 385

Wright, A. N., Longbottom, A. W., Mills, K. J., \& Ruderman, M. S. 2002, J. Geophys. Res., 107, 1242 